Chirurg 2020 $91: 265-266$

https://doi.org/10.1007/s00104-020-01120-y

Online publiziert: 17. Februar 2020

(c) Der/die Autor(en) 2020

\section{JünDNIS Ärzte}

Bündnis Junge Ärzte

\title{
Positionspapier des Bündnis Junge Ärzte (BJÄ) zu Digitalisierung, Applikationen (Apps) und Künstlicher Intelligenz im Gesundheitswesen
}

landes- und bundespolitisch Verantwortlichen - und nicht das der Ärzte - der Hemmschuh in puncto Digitalisierung.

Das Bündnis Junge Ärzte fordert deshalb die Politik auf, ihrer Investitionsverpflichtung im Gesundheitswesen nachzukommen, damit schon längst mögliche Innovationen in den klinischen Alltag Einzug halten.

Der demographische Wandel in Deutschland sowie die zunehmende Komplexität der Medizin, aber auch die zunehmend eingeschränkte fachärztliche Versorgung ländlicher Regionen erfordern den Ausbau der digitalen Infrastruktur, bringen zugleich aber auch viele Innovationen in die Versorgung. Basierend auf einer modernen IT-Infrastruktur wünschen wir uns, dass zukünftig Anwendungen und Applikationen unseren Arbeitsalltag erleichtern. Diese können die Ärzteschaft unter anderem unterstützen, die wachsenden Dokumentationsansprüche effizient $\mathrm{zu}$ bewältigen oder Routinearbeit sinnvoll $\mathrm{zu}$ stratifizieren. Es können dringliche Befunde und Entscheidungen priorisiert werden und eine verbesserte Versorgungsqualität erreicht werden. Die Integration künstlicher Intelligenz (KI) in den klinischen Alltag ist für uns wichtig - die Auswertung von großen Datenmengen kann neue wissenschaftliche Erkenntnisse generieren. Auch bietet KI-unterstützte Diagnostik als Berater des behandelnden Arztes das Potential die Patientenversorgung in Teilgebieten zu verbessern. Wir sind offen für diesen Wandel - er soll und muss allerdings durch uns Ärzte mitgestaltet werden und daher fordern wir eine frühe Einbindung der Ärzteschaft und insbesondere der jungen Ärzte in die Entwicklung von digitalen Produkten.

Gleichzeitig sehen wir auch die Kehrseiten des digitalen Wandels: Es darf nicht dazu kommen, dass vermeintlich sinnvolle Applikationen nicht dem Interesse unserer Patienten, sondern vornehmlich den Interessen Dritter (wie Investoren, Krankenkassen oder dem Staat) dienen. Eine Datenspende, z.B. zum Ausbau der Versorgungsforschung und zur Entwicklung intelligenter medizinischer Anwendungen erachten wir in geordnetem Setting für sinnvoll. Die im klinischen Alltag erhobenen Daten sollten für die Forschung nutzbar gemacht werden und es sollte ein gesetzlicher Rahmen für den Austausch unter Wahrung höchstmöglicher Datensicherheit geschaffen werden. Es muss insbesondere transparent bleiben, wann und wo von Patienten und Ärzten sensible Behandlungsdaten an Dritte weitergeben werden. Wir fordern, dass nur Anwendungen und Applikationen Einzug in die Versorgung halten, die der Versorgungssicherung bzw. der Versorgungsqualität unserer Patienten dienen. Zudem muss darauf geachtet werden, dass - beispielsweise beim Einsatz von $\mathrm{KI}$ - medizinische Entscheidungen nachvollziehbar und transparent bleiben, um die hohe Akzeptanz unseres Gesundheitswesens in der Bevölkerung nicht zu gefährden. Nur wenn die Ärzteschaft eine maßgebliche Rolle in der Entwicklung traktivität Deutschlands als Forschungs-
standort. Aktuell ist das Verhalten der 
von digitalen medizinischen Produkten einnimmt, kann sie als Garant für die Einhaltung des Patienteninteresses eintreten, so dass medizinische Entscheidungen verantwortbar und auch justiziabel bleiben.

Um den Herausforderungen der nächsten Jahrzehnte gerecht zu werden,

"equally contributed authors": Max Tischler JuDerm:Junge Dermatologen im Berufsverband derDeutschen Dermatologene. V.; Mira Faßbach und Carolin Siech - Germans society of residents in Urology e.V., Assistenzärzte der Urologie; Tobias Fritz-Deutsche GesellschaftfürChirurgie e. V.; Benedikt Braun - Berufsverband der Deutschen Chirurgen e. V.; Kevin Schulte und Cornelius Weiß-Junges Forum des Berufsverbandes deutscher Internisten e. V.; Hanna Josephin Eisenberg und Samuel Knauß - Deutsche Gesellschaft für Neurologie - Junge Neurologen der DGN e. V.; Clara Matthiessen und Lukas Fricker - Junge Internisten - Arbeitsgruppe der Deutschen Gesellschaft für Innere Medizin; Marc Riedling - Berufsverband Deutscher Anästhesisten e. V.; Chris Sommer - Berufsverband der Ärzte für Mikrobiologie, Virologie und Infektionsepidemiologie e. V.; Malte Sieren - Deutsche Röntgengesellschaft e.V.; Anna Maria Schuh und Johannes Birtel - Young DOG der Deutsche GesellschaftfürOphthalmologie;Ahmad Zhaour - Berufsverband der Augenärzte Deutschlands e. V.;Anna-Katharina Doepferund Stefanie Weber - Junges Forum OundU, Deutsche Gesellschaft und Berufsverband für Orthopädie und Unfallchirurgie; Iris Eckhardt - Deutsche Gesellschaft für Kinder- und Jugendmedizin e. V.; Max Kittel und Larissa Lauterbach - Junges Labor in der Deutschen Gesellschaft für Klinische Chemie und Laboratoriumsmedizin e.V.; Anna McLean, Stefanie Maurer und Meriem Makina - Deutsche Gesellschaft für Neurochirurgie; Murat Yilmaz und Sami Shabli - HNO-Assistenten, Deutsche Gesellschaft für Hals-, Nasen-, Ohrenheilkunde, Kopf-, und Halschirurgie e.V.; Helena Radbruch - AG Junge PathologInnen des Bundesverbands Deutsche Pathologen; Markus Haun und Katharina Hof - Junges Forum in der Deutschen Gesellschaft für Psychosomatische Medizin und Ärztliche Psychotherapie; Juliane Kröplin - Deutsche Gesellschaft für Mund-, Kiefer- und Gesichtschirurgie; Vera Hepp und Martin Weiß - Junges Forum der Deutschen Gesellschaft für Gynäkologie und Geburtshilfe e. V.; Sandy Pistol - Deutsche Gesellschaft für Psychiatrie und Psychotherapie, Psychosomatik und Nervenheilkunde e. V.

Hinweis zu geschlechterneutraler Sprache: Aus Gründen der leichteren Lesbarkeit wird in dem vorliegenden Artikel überwiegend die gewohnte männliche Sprachform verwendet. Dies impliziert jedoch keine Benachteiligung eines anderen Geschlechts, sondern soll im Sinne der sprachlichen Vereinfachung als geschlechtsneutral zu verstehen sein. brauchen wir die digitale Unterstützung und weitere strukturelle Veränderungen im Gesundheitswesen. Darüber hinaus fordern wir, dass digitale Kompetenzen in der Aus- und Weiterbildung von Medizinern einen größeren Stellenwert einnehmen. Nur so kann der behandelnde Arzt seiner Funktion als Lotse und Partner des Patienten auch in Hinblick auf technische bzw. digitale Innovationen gerecht werden. Bereits in der Versorgung befindliche Ärzte müssen entsprechend geschult werden, ohne dass ihnen Kosten entstehen. Der Arbeitsalltag der Ärzte muss und wird sich verändern. Der Anspruch der Patienten nach fachlich korrektem Handeln, aber auch einfühlsamer und persönlicher Aufklärung und Beratung bleibt jedoch Basis der medizinischen Behandlung. Daher stehen wir einem übermäßigen Ausbau der Telemedizin, insbesondere im Erstkontakt mit dem Patienten, weiterhin zurückhaltend gegenüber. Zielgerichteter Einsatz telemedizinischer Anwendungen allerdings, etwa die teleradiologische Versorgung peripherer Krankenhäuser, kann die Patientenversorgung aber verbessern. Hier ist eine gesetzliche Grundlage notwendig, um zu garantieren, dass entsprechende Dienstleistungen die hohen Qualitätsstandards des deutschen Gesundheitswesens erfüllen. Die digitalen Innovationen müssen weiterhin der Intimität des Arzt-Patienten-Kontakts gerecht werden und dürfen diese nicht verdrängen. Das Arzt-Patienten-Verhältnis muss auch in Zukunft das Rückgrat des deutschen Gesundheitswesens bleiben.

Berlin, den 09.12.2019

\section{Korrespondenzadresse}

\section{Dr. med. Tobias Fritz}

Klinik für Unfall-, Hand- und Wiederherstellungschirurgie, Universitätsklinikum des Saarlandes

Kirrbergerstr. 100, Geb. 57, 66421 Homburg/

Saar, Deutschland

Tobias.fritz@uks.eu

Funding. Open Access funding provided by Projekt DEAL.

\section{Einhaltung ethischer Richtlinien}

Interessenkonflikt. T. Fritz gibt an, dass kein Interessenkonflikt besteht.

Für diesen Beitrag wurden von den Autoren keine Studien an Menschen oder Tieren durchgeführt. Für die aufgeführten Studien gelten die jeweils dort angegebenen ethischen Richtlinien.

Open Access. Dieser Artikel wird unter der Creative Commons Namensnennung 4.0 International Lizenz veröffentlicht, welche die Nutzung, Vervielfältigung, Bearbeitung, Verbreitung und Wiedergabe in jeglichem Medium und Format erlaubt, sofern Sie den/die ursprünglichen Autor(en) und die Quelle ordnungsgemäß nennen, einen Link zur Creative Commons Lizenz beifügen und angeben, ob Änderungen vorgenommen wurden.

Die in diesem Artikel enthaltenen Bilder und sonstiges Drittmaterial unterliegen ebenfalls der genannten Creative Commons Lizenz, sofern sich aus der Abbildungslegende nichts anderes ergibt. Sofern das betreffende Material nicht unter der genannten Creative Commons Lizenz steht und die betreffende Handlung nicht nach gesetzlichen Vorschriften erlaubt ist, ist für die oben aufgeführten Weiterverwendungen des Materials die Einwilligung des jeweiligen Rechteinhabers einzuholen.

Weitere Details zur Lizenz entnehmen Sie bitte der Lizenzinformation auf http://creativecommons.org/ licenses/by/4.0/deed.de.

\section{Literatur}

1. WHO (2016) From innovation to implementation. ehealth in the WHO European region. http:// www.euro.who.int/_data/assets/pdf_file/0012/ 302331/From-Innovation-to-ImplementationeHealth-Report-EU.pdf?ua=1. Zugegriffen: 17. Nov. 2019 